Integrating the light and dark sides of student engagement

using person-oriented and situation-specific approaches 


\begin{abstract}
This study contributes to the research on student engagement in three ways: 1) by combining questionnaire and situational measures of engagement using the Experience Sampling Method (ESM), 2) by applying a demands-resources model to describe the positive and negative aspects of student engagement, and 3) by adopting a person-oriented approach to describe subgroups of students with different profiles of engagement and burnout symptoms. Two studies were conducted: sample one comprised 255 US high school students $\left(45.5 \%\right.$ female, $9^{\text {th }}-12^{\text {th }}$ grade), and sample two 188 Finnish comprehensive and high school students (59.6\% female, $9^{\text {th }}$ to $10^{\text {th }}$ grade). Latent profile analyses (LPA) of person-level measures of schoolwork engagement and burnout in the US and Finland revealed four profiles: 1) engaged, 2) engaged-exhausted, 3) moderately burned out (risk for burnout) and 4) burned out. These four groups were identified in both samples, but differed in their prevalence. The groups differed in their state experiences of situational demands, resources and engagement at school. Engagement is not wholly an experience of 'flourishing': some students experienced elevated levels of both engagement and burnout. Thus, positive and negative aspects of engagement should be studied and modeled together.
\end{abstract}

Keywords: Schoolwork Engagement, School Burnout, Demands-Resources Model, PersonOriented Approach, Situational Measures 


\section{Integrating the light and dark sides of student engagement using person-oriented and situation-specific approaches}

This study describes three new directions in the research on schoolwork engagement (Salmela-Aro \& Upadyaya, 2012) and burnout (Salmela-Aro, Kiuru, Leskinen, \& Nurmi, 2009a). First, we introduce situation-specific measures of malleable aspects of engagement. Second, we describe the demands-resources model applied to the present research on student engagement, emphasizing how insightful and necessary it is to analyze the positive aspects of engagement along with exhaustive experiences and symptoms of school-related burnout. Finally, we show how new insights can be gained through person-oriented analyses combining engagement and burnout.

\subsection{Integrating positive and negative aspects of engagement in a demands-resources model}

Student engagement is a multifaceted construct that describes malleable aspects of motivation and behavior that are beneficial for learning and adjustment in the school context. This broad concept comprises emotional, cognitive and behavioral aspects. The present study mainly focuses on the emotional engagement. Emotional engagement can be defined as student reactions to teachers, classmates, and school-related experiences, including, among others, affect, identification, belonging, and value (Fredricks, Blumenfeld, \& Paris, 2004; Wang \& Degol, 2014). For instance, some researchers have described emotional engagement as a student's feeling that learning in school is essential and interesting and that school education is an instrument for achieving personal goals (Finn, 1989; Voelkl, 1997). Wang and Peck (2013) measured emotional engagement defined as happiness and interest and found that students with low emotional but high cognitive and behavioural engagement suffered increased rates of depression compared with peers. Intrinsic and emotional experiences are emphasized in the definition of schoolwork engagement used in the present study: Salmela-Aro and Upadyaya (2012) adapted measures of work engagement to the school context, defining schoolwork engagement as the experience of energy, dedication and absorption at school. Whereas energy refers to feeling energetic, active and vigorous when doing 
school-related tasks, dedication describes the experience of school-related work as meaningful and inspiring, and absorption describes experiences of deep involvement, concentration and working intensively applied in this study.

While engagement is often described as a positive, 'flourishing' experience (Reschly et al., 2008), several researchers have pointed out that, in order to achieve a holistic explanation for relevant outcomes, engagement should also be considered in combination with negative experiences. The emotional engagement literature distinguishes between positive emotional student engagement (e.g. interest, happiness, joy) and negative disengagement (e.g. anxiety, sadness, stress), and it has been shown that both constructs are empirically distinct and contribute uniquely to the explanations of relevant outcomes (Jimerson, Campos, \& Greif, 2003; Skinner, Furrer, Marchand, \& Kinderman, 2008). In line with this distinction between positive and negative aspects of emotional engagement, the demands-resources model describes two different psychological processes: a positive process of resources leading to engagement, positive outcomes and well-being, and a negative process of demands leading to strain, disengagement, and symptoms of exhaustion and even burnout (Salmela-Aro \& Upadyaya, 2014; see also Bakker \& Demerouti, 2007). Recent studies have adapted the demands-resources model to the study of disengagement and burnout in the school context, defining burnout as a feeling of exhaustion and indifference towards schoolrelated work, and feelings of inadequacy as a student (Salmela-Aro et al., 2009a). School burnout describes a continuous process of feeling overwhelmed, lacking enjoyment and seeing little value in school-related work tasks and has been found to predict later decline in schoolwork engagement, impaired well-being and maladjustment at school (Salmela-Aro \& Upadaya, 2012; 2014). The negative emotional processes described by the construct of school burnout resemble those of the concept of emotional disengagement in school. Both low emotional engagement and school burnout are linked to symptoms of depressive symptoms (Salmela-Aro \& Upadyaya, 2014). A longitudinal cross-lagged study revealed that school burnout predicted depressive symptoms rather than vice 
versa (Salmela-Aro et al., 2009b). In the present study, we use the construct of school burnout to describe the maladaptive characteristics of negative emotional processes in school, and the construct of schoolwork engagement to describe adaptive positive emotional processes in school.

The demands-resources model suggests that study-related resources are the most important predictors of schoolwork engagement, while study-related demands are central determinants of school burnout (Salmela-Aro \& Upadyaya, 2014). We define study demands as what the school requires students to do or to achieve, the challenges that motivate students to invest effort, and the emotional costs that result from the perceived necessity to meet such expectations. Study resources are defined as the feeling that one is able to keep up with the demands and challenges of studying and meeting one's own learning goals and awareness of and confidence in one's level of skills. Thus, study resources refer to 'being able to', while study demands refer to 'being required to'. Personal resources, such as self-efficacy beliefs, facilitate schoolwork engagement and help students to meet their study targets and goals. Study resources are positively correlated with engagement, and study demands are positively correlated with school burnout (Salmela-Aro \& Upadyaya, 2013).

\subsection{Situational measures and fluctuations of student engagement}

Although student engagement is conceptualized as malleable (Fredricks, Blumenfeld, \& Paris, 2004), it has mostly been assessed with rather stable measures (Fredricks \& McColskey, 2012). Using such scales, most research on engagement has focused on the differences between individuals and has treated day-to-day and moment-to-moment fluctuations in engagement and burnout as measurement error. The typical self-report questionnaires measuring engagement require individuals to aggregate in their own minds how they feel about school generally, that is, across a whole range of experiences. This approach is not only biased by student response bias and memory errors, but is also of little use in studying the changeable determinants of student engagement, which are of paramount interest to teachers. While it is informative to know students' levels of 
enduring school engagement and burnout, analysis of daily and situation-specific changes can inform of the reasons why these experiences change between situations, days, and contexts, and what teachers can do to promote their students' engagement.

One such approach is to gather repeated measurements of situational engagement in a representative sample of everyday life activities, for instance with smartphone applications that trigger short questionnaires several times a day (experience sampling method, ESM). ESM reduces response bias (Goetz et al., 2013) and memory recall errors because it assesses experiences at the moments in which they occur. ESM also allows investigation of the influence of the context and other situational and short-term determinants, which helps to identify activities or environments that are particularly engaging, or exhaustive, effortful and frustrating (i.e. disengaging). Studies utilizing situational measures found daily fluctuations in engagement (Petrou et al., 2012; Simbula, 2010), which indicates the necessity to study these malleable aspects with state measures in greater detail. Previous ESM studies have assessed situational engagement with state measures tapping situational interest, enjoyment, and concentration (Shernoff \& Vandell, 2007). Other researchers have used items assessing current skills (= resources) and current challenges (= demands) to assess states of engagement (e.g. Csikszentmihalyi \& Schneider, 2000). Feeling active and in control of a current activity were also found to be important aspects of emotional engagement at school (Engeser \& Rheinberg, 2008; Pekrun, Elliot, \& Maier, 2009; Shernoff et al., 2003). Disengaging and demanding moments can be assessed by asking students how stressed, anxious and bored they feel (Skinner et al., 2008).

\subsection{School Engagement and School Burnout in Finland and the US}

Our understanding of school engagement is limited by the fact that most of the research on emotional engagement at school has focused on adolescents in the United States. Therefore, this paper examines emotional engagement in two samples, one from the US and one from Finland. The Finnish culture values education and academic achievement, and Finnish schools employ 
RUNNING HEAD: Integrating the light and dark side of student engagement.

heterogenous grouping, the practice of keeping students of all ability levels in the same classroom. In Finland, students attain consistently high levels of academic achievement throughout secondary school (OECD, 2013). Less is known about their emotional engagement. The 2012 PISA survey found that Finland and the US ranked below the OECD average in terms of how many 15-year-old students felt happy at school. In the 65 -country comparison, the US was ranked $48^{\text {th }}$, with almost $80 \%$ of students in the category happy, and Finland $60^{\text {th }}$, with less than $70 \%$ of students classified as happy (OECD, 2013). In addition, there is mounting evidence that Finnish students also have negative feelings about school (Linnakyla \& Malin, 2008; Tynkkynen \& Salmela-Aro, 2012). For instance, Salmela-Aro et al. (2009a) found that many Finnish secondary school students felt inadequate as a student, exhausted by school, and cynical about the value of school, feelings that we understand as indicators of disengagement and burnout. However, compared to students in Belgium, Italy, Germany, the Netherlands and Portugal, Finnish students reported relatively high levels of schoolwork engagement and low levels of school burnout (Kinnunen et al., in prep.). Compared to US students, Finnish students experienced more moments of situational engagement (defined as states characterized by high skill, challenge, and interest) at school (13.0\% in the US'. versus $17.0 \%$ in Finland; Schneider et al., in press).

\subsection{Variable- and person-oriented approaches}

The previous research has predominantly applied variable-oriented approaches: co-variances between engagement, disengagement, and the relevant outcome variables on the population level (see Roorda, Koomen, Spilt, \& Oort, 2011). These variable-oriented approaches have mostly found that (1) engagement and disengagement are negatively correlated with each other cross-sectionally and over time (e.g. Salmela-Aro \& Upadyaya, 2014); (2) engagement is predicted by personal resources, while disengagement and burnout are predicted by study demands (Salmela-Aro \& Updyaya, 2014); and (3) engagement predicts desired outcomes such as well-being and persistence 
in educational pathways, while disengagement and burnout predict undesired outcomes such as the development of depressive symptoms or school drop-out (Salmela-Aro \& Updyaya, 2014).

However, such variable-oriented approaches describe overall tendencies between variables, behind which differently functioning subgroups of individuals may be concealed (Bergman \& Magnusson, 1997; Reizle, 2013). When engagement and burnout are analyzed together with person-oriented approaches, a different picture emerges for some students. For instance, analyzing subgroups of individuals with latent profile analyses, Tuominen-Soini and Salmela-Aro (2014) found that up to one in four Finnish high school students experienced above-average levels of both engagement and exhaustion simultaneously (engaged-exhausted group), while the three other groups were (1) engaged, (2) cynical (moderate burnout) and (3) burned out. The engagedexhausted individuals were likely to develop depressive symptoms longitudinally. The finding of students with elevated engagement and burnout levels challenges the common idea of engagement as a wholly positive experience. This is easily overlooked if the analysis is limited to the correlations between engagement, disengagement and relevant outcomes, and it is also the unique contribution of applying a person-oriented approach.

\subsection{Research Objectives and Hypotheses}

The present study aimed to integrate (a) situation-specific measures of emotional engagement, (b) a demands-resources perspective applied to the school context, and (c) person-oriented analysis of profiles of emotional engagement and school burnout among Finnish and US adolescents. The research objectives refer to combinations of these three new aspects.

Our first research objective was to explore associations between questionnaire scales measuring schoolwork engagement and burnout and in-the-moment measures of situational demands, resources, and emotional engagement in the school context. Here, we expected that our analyses would yield positive correlations between a student's schoolwork engagement, their situational 
resources, and their engagement experiences at school. We also expected that school burnout would correlate positively with a student's average level of situational demands experienced at school.

Our second research objective was to explore the demands-resources model of schoolwork engagement and burnout using person-oriented statistical methods. For these analyses, we used a kind of person-oriented modeling technique called Latent Profile Analysis. We used this LPA method to identity characteristically distinct sub-populations of engagement and burnout. We then compared results from these analyses to those yielded from a different population of Finnish students (i.e., Tuominen-Soini and Salmela-Aro, 2014).

Our third and final research objective was to explore the extent to the engagement patterns of Finnish students might be reflected in other countries and contexts. Here, we used the LPA approach to examine the extent to which the results yielded from our Finnish sample might be replicated using data drawn from a sample of students from the United States. These results provide unique comparative insight into the promises and pitfalls of student engagement across contexts.

\section{METHODS}

\subsection{General procedure}

We used data from the EAGER study, a collaborative research project conducted in 2013 and 2014 in Finland and the US. From the 517 participants, 487 responded to the schoolwork engagement and school burnout scales. In addition we assessed their situational experiences with an ESM procedure. The students were given smartphones with an application that prompted eight short questions a day over seven consecutive days, where each question was indicated by a short acoustic 
RUNNING HEAD: Integrating the light and dark side of student engagement.

signal, and the students were instructed to answer immediately after hearing the signal. Most of the ESM questions were administered at random intervals between eight a.m. and eight p.m., but owing to the EAGER study's focus on science engagement, in each science lesson fixed time intervals were used. Since we were interested in school engagement and burnout, we only analyzed ESM data collected during the students' school days, using the students' official lesson schedules to determine whether a given beep had occurred during the student's school day or outside of school. All the ESM data analyses are based on the responses of individuals who answered at least four ESM questions in school or questions on all the target experiences.

The US data were collected in four rural and urban high schools in Eastern Michigan from 255 students $\left(45.5 \%\right.$ female; $539^{\text {th }}$ graders, $11910^{\text {th }}$ graders, $3411^{\text {th }}$ graders, $3012^{\text {th }}$ graders; 5,022 ESM in-school questions, mean per person 18.3 questions).

The Finnish data were collected in two comprehensive and two high schools in the Finnish capital Helsinki from 188 students $\left(59.6 \%\right.$ female; $1029^{\text {th }}$ graders and $7310^{\text {th }}$ graders; 3,174 ESM in-school questions, mean per person 16.6 questions).

\subsection{Measures}

The schoolwork engagement inventory (SEI; Salmela-Aro \& Upadyaya, 2012) was developed based on measures of work engagement (Bakker \& Demerouti, 2007). This 9-item scale measures the following three sub-facets of school-related engagement: energy ( 3 items, "At school I am bursting with energy."), absorption (3 items, "Time flies when I am studying.") and dedication (3 items: "I find schoolwork full of meaning and purpose."). The original response scale taps how often the indicators of school engagement are experienced, from $1=n e v e r$ to $7=$ daily. In 109 individuals in the Finnish sample, the SEI was assessed on a response scale from $1=$ not at all to 4 $=$ very much . In order to convert all measures to the same metric, the answers were transformed with the monotonic 'proportion of maximum scaling' (POMS) method (Little, 2013). The transformed scale ranged from $0=$ lowest score to $1=$ highest score. As all the items have been 
RUNNING HEAD: Integrating the light and dark side of student engagement.

found to load on one and the same factor of student engagement in previous studies (Salmela-Aro \& Upadyaya, 2012), we used the mean composite score of all nine items as a measure of schoolwork engagement.

The school burnout inventory (SBI) is based on prior research on job burnout and the definition of burnout as a syndrome comprising emotional exhaustion, cynicism, and inadequacy (Salmela-Aro et al., 2009a). This scale measures three facets of school-related burnout, exhaustion (4 items, "I feel overwhelmed by my schoolwork.”), cynicism (3 items, "I’m continually wondering whether my schoolwork has any meaning.") and inadequacy (2 items, "I often have feelings of inadequacy in my schoolwork."). The response scale for the SBI ranges from 1=Strongly Disagree to 6=Strongly Agree. The answers were therefore transformed with the POMS method (see above). The three-factor structure has been supported in prior studies (Salmela-Aro et al., 2009), and therefore we used the three subscales as measures of school burnout.

ESM measures of situational resources, demands, and engagement indicators

Situational resources were assessed with four items asking how skilled, confident, successful and in control the individual felt at the time of the ESM signal. Situational demands were assessed by asking how stressed, anxious, confused, and challenged the individual felt at the moment of the ESM signal Likert scale ranging from $1=$ not at all/low to $4=$ very $\mathrm{much} / \mathrm{high}$. Situational engagement were assessed with four ESM items tapping how active and interested the individuals felt in the present activity, whether they were enjoying what they were doing and how important the current task was to them. The situational resources, demands, and engagement items had good reliabilities of $.68 \leq \alpha \leq .81$ (school beeps of individuals with at least four in-school beeps). The item-construct assignment was also supported in the subsequent exploratory and twolevel confirmatory factor analyses (Table 2; inter-correlations Table A-1).

\subsection{Analyses}


RUNNING HEAD: Integrating the light and dark side of student engagement.

First, we examined descriptives (M, SD, ICC, country differences in mean levels) and the measurement properties of the applied scales and items, including internal consistencies and confirmatory factor analyses. The descriptives for the situational ESM measures were derived from multilevel models with ESM beeps (= within-level) nested in individuals (= between-level).

Variable-oriented approaches within a demands-resources model of schoolwork engagement and school burnout

Second, we tested whether with variable-oriented approaches we could replicate the previous findings, which had shown that schoolwork engagement was a mainly positive experience, and school burnout was a mainly negative experience.

We analyzed whether the SEI and the SBI were negatively correlated with each other in Finland and in the US, and we analyzed how the SEI and the SBI correlated with individuals' average levels of situational demands, resources, and engagement experienced at school. Since the ESM data were nested (within-level = situations, between-level = individuals), we computed the between-level correlations between the SEI, SBI and person-average in-school ESM experiences of demands, resources and engagement in a two-level model in Mplus (version 7.1; Muthén \& Muthén, 1998-2012), where the ESM items were allowed to vary on the within-level. Only individuals with at least four beeps in the within-school context were included in these analyses.

\section{Person-oriented approaches within a demands-resources model of engagement and burnout}

We expected that person-oriented analyses of the SEI and SBI would contribute insights that might be overlooked with the variable-oriented approaches. These expectations were based on earlier findings by Tuominen-Soini and Salmela-Aro (2014), who had identified a subgroup of individuals with above-average levels of both schoolwork engagement and school burnout. Since these findings were reported only for Finnish students with data obtained ten years earlier, we were interested in learning whether similar groups would be found in current samples from Finland and 
the US, and how these groups would differ in terms of their experiences of situational demands, resources, and school engagement criteria.

To identify eventual subgroups of individuals with distinct engagement-burnout profiles, we conducted latent profile analyses (LPA), using the schoolwork engagement score and the three school burnout subscales as indicators. These indicators were chosen because they had been used in the study we aimed to replicate (Tuominen-Soini \& Salmela-Aro, 2014), and because this structure of SEI and SBI measures has previously been supported (Salmela-Aro \& Upadyaya, 2012).

Because of the relatively small sample size, we entered these indicators as manifest items (mean scores of the corresponding items) in the LPA. The LPA were computed in Mplus 7.1. In order to find out whether similar groups existed in both countries, we conducted a multi-group LPA (KNOWNCLASS command) estimating the profiles simultaneously in both countries. A robust estimator (MLR) was chosen.

Models with two, three, four, five, and six profiles were estimated with LPA (first separately for each country, then simultaneously in the multi-group LPA), and then compared on the following model fit criteria: 1) the log likelihood that each model had been replicated; 2) models with smaller AIC, BIC, CAIC and AWE (indicators of model fit and parsimony) preferred over models with larger values for these criteria; 3) the Bayes Factor and Correct Model Probability (see Masyn, 2013) were used to identify the most likely correct model among the set of estimated models; and 4) models with better overall classification of individuals into profiles, as indicated by larger entropies, were preferred over models with smaller entropies. As a more specific criterion for the classification of individuals into profiles, we examined the average latent class probability for the most likely latent class membership by latent class, which in a good model should not drop below 0.8 at the main diagonal (Rost, 2006). Finally, we considered the criteria of interpretability, parsimony, and problem-free estimations, meaning that if there was no further change in the interpretation when more profiles were added, then the most parsimonious model was preferred. 
RUNNING HEAD: Integrating the light and dark side of student engagement.

For the sake of parsimony, models with fewer classes were preferred over models with a higher number of classes, if this was not in conflict with any of the afore-mentioned criteria. The LoMendell-Rubin Likelihood Ratio Test and the Bootstrapped Likelihood Ratio Test were used to discard models that did not fit the data better than a more parsimonious model. These tests were applied in the country-specific LPA, but were not available for the final multi-group comparison (via KNOWNCLASS command).

The profiles identified with the person-level LPAs were then compared to individuals' average levels of in-school experienced situational demands, resources and engagement criteria. For this purpose, we first computed each person's average level of situational demands, resources and engagement across all the ESM beeps that had occurred at school, and then, using MANOVAs, compared whether the profiles (obtained from the LPA) differed in their profile averages of these person-level means of situational in-school demands, resources, and engagement.

\section{Replication in two samples (Finland, US)}

In order to explore whether these results would be replicate in the two samples, we conducted all the analyses separately for the Finnish and US samples (and the LPA parallel in each country). To facilitate comparison, the results for the US and Finnish students are reported next to each other in the same tables.

\section{RESULTS}

\subsection{Descriptives and Measurement Properties}

The SEI and the subscales of the SBI were sufficiently reliable in both the Finnish and US sample. Mean engagement, cynicism and feeling of inadequacy differed significantly between the Finnish and US samples (Table 1). The Finnish students reported higher engagement (small effect size), while the US students reported higher cynicism (large effect size) and stronger feelings of inadequacy (small effect size). 


\section{Table 1}

Descriptives and country differences for the SEI and SBI

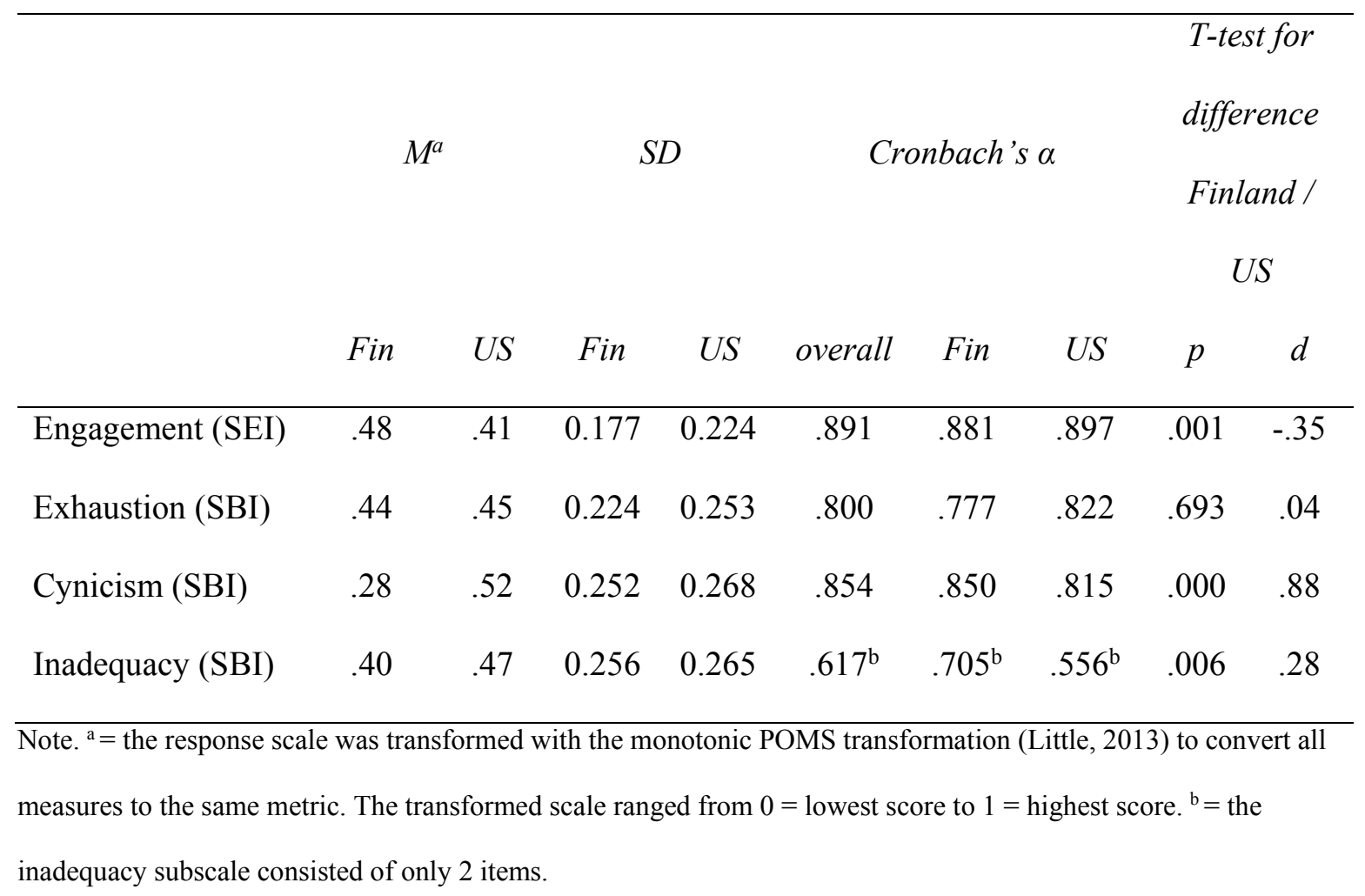

Previous studies have found a one-factor structure for the schoolwork engagement inventory (Salmela-Aro \& Upadyaya, 2012). Therefore we tested the fit of a model where all the SEI items loaded on a common factor. For the school burnout inventory, a structure with three correlated subscales (exhaustion, cynicism and inadequacy) was found in earlier studies (Salmela-Aro et al., 2009a) and was therefore expected in the present one. In the Finnish sample, the confirmatory factor analyses for both the schoolwork engagement and burnout fitted the data satisfactorily [Schoolwork engagement: $X^{2}(\mathrm{df})=61.215(27) ; \mathrm{p}=.000$; RMSEA $=.078$; C.I.: .052 - .104; CFI $=$ $.963 ; \mathrm{TLI}=.950 ;$ SRMR $=.036 ;$ School burnout: $X^{2}(\mathrm{df})=57.830(24) ; \mathrm{p}=.000 ; \mathrm{RMSEA}=.082 ;$ C.I.: $.055-.109 ; \mathrm{CFI}=.964, \mathrm{TLI}=.946 ; \mathrm{SRMR}=.044]$. In the US sample, the confirmatory factor analyses for both scales yielded only moderately satisfactory model fits due to correlated residuals [Schoolwork engagement: $X^{2}(\mathrm{df})=139.388(27) ; \mathrm{p}=.000$; RMSEA = .130; C.I.: .109 - .151; CFI $=$ 
$.903 ; \mathrm{TLI}=.871 ; \mathrm{SRMR}=.049 ;$ School burnout: $X^{2}(\mathrm{df})=113.480(24) ; \mathrm{p}=.000 ; \mathrm{RMSEA}=.123$;

C.I.: $.101-.146 ; \mathrm{CFI}=.913, \mathrm{TLI}=.869 ; \mathrm{SRMR}=.052]$.

Descriptives for situational experiences of demands, resources and engagement in school

The situational measures of study demands, resources, and engagement were reliable and supported by confirmatory factor analyses (see Table 2). For ICCs, between-level means, and within- and between-level variances of situational demands, resources, and engagement (Table A-2 appendix). 


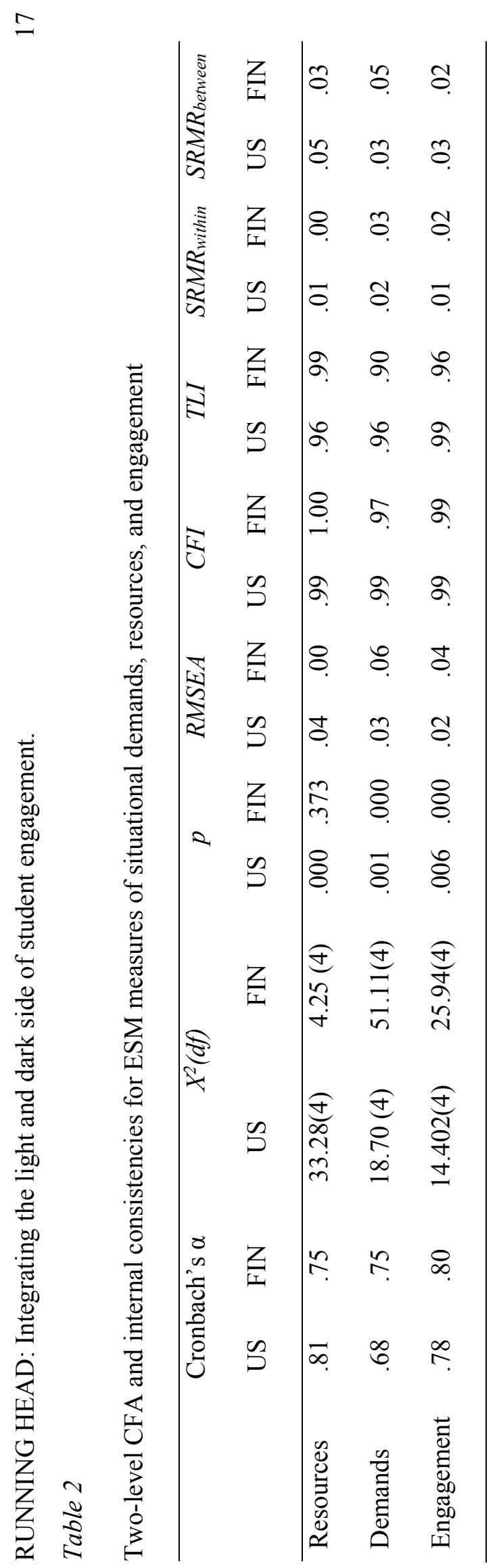




\subsection{Variable-oriented Approach}

The results showed that (Table 3) schoolwork engagement was weakly negatively correlated with the subscales of the school burnout inventory in the US sample $(-.165 \leq \mathrm{r} \leq-.206, \mathrm{p}<.05)$. In Finland, only the cynicism subscale was significantly related to the schoolwork engagement score ($.224, \mathrm{p}<.05)$.

Moreover, schoolwork engagement was positively correlated with individuals' average level of situational resources and engagement experienced within school, and unrelated to situational demands, in both countries. Also the three subscales of the school burnout were positively correlated with individuals' average level of in-school experienced demands in both countries. Moreover, the three burnout subscales correlated negatively with the individual's average level of resources in the US, but were unrelated to resources in Finland. While the correlations point in the expected directions, their effect sizes are small.

\section{Table 3}

Correlations of the person-level (= between-level) of Schoolwork Engagement (SEI) and School Burnout (SBI) with the individuals' mean scores of in-school situational demands, resources, and engagement (ESM)

\begin{tabular}{lcccccccc}
\hline $\begin{array}{l}\text { person-level } \\
\text { average of ESM }\end{array}$ & \multicolumn{2}{c}{$\begin{array}{l}\text { Schoolwork } \\
\text { Engagement }\end{array}$} & \multicolumn{2}{c}{ Exhaustion } & \multicolumn{2}{c}{ Cynicism } & \multicolumn{2}{c}{ Inadequacy } \\
measure at school & USA & FIN & USA & FIN & USA & FIN & USA & FIN \\
\hline Resources & $.247^{*}$ & $.409^{*}$ & $-.243^{*}$ & -.052 & $-.212^{*}$ & -.190 & $-.244^{*}$ & -.036 \\
Demands & -.020 & -.146 & $.345^{*}$ & $.486^{*}$ & $.227^{*}$ & $.440^{*}$ & $.375^{*}$ & $.411^{*}$ \\
Engagement & $.361^{*}$ & $.368^{*}$ & -.068 & .087 & $-.217^{*}$ & -.047 & -.100 & .088 \\
\hline
\end{tabular}

Note. $*: \mathrm{p} \leq .05$.

\subsection{Person-oriented Approach}

\section{Replication of engagement and burnout profiles with person-level latent profile analyses (LPA)}

Table 4 shows that the results of the multi-group LPA. The BIC and the Correct Model Probability favoured the model with three and the model with four profiles, while the AIC and CAIC supported 
the models with the highest number of profiles. The Bayes Factor indicated moderate evidence for the three and four-profile models, and strong evidence against the six-profile model.

The four-profile model was the common denominator of the best fits in BIC, Correct Model Probability, and Bayes Factor. Since entropy was high (above .80), we were confident in using the MANOVA (Ram \& Grimm, 2009). This model also resembled the solution previously described by Tuominen-Soini \& Salmela-Aro (2014), replication of which we were also interested in exploring in the present samples. These were the reasons why we decided to examine the model with four profiles more in detail. 


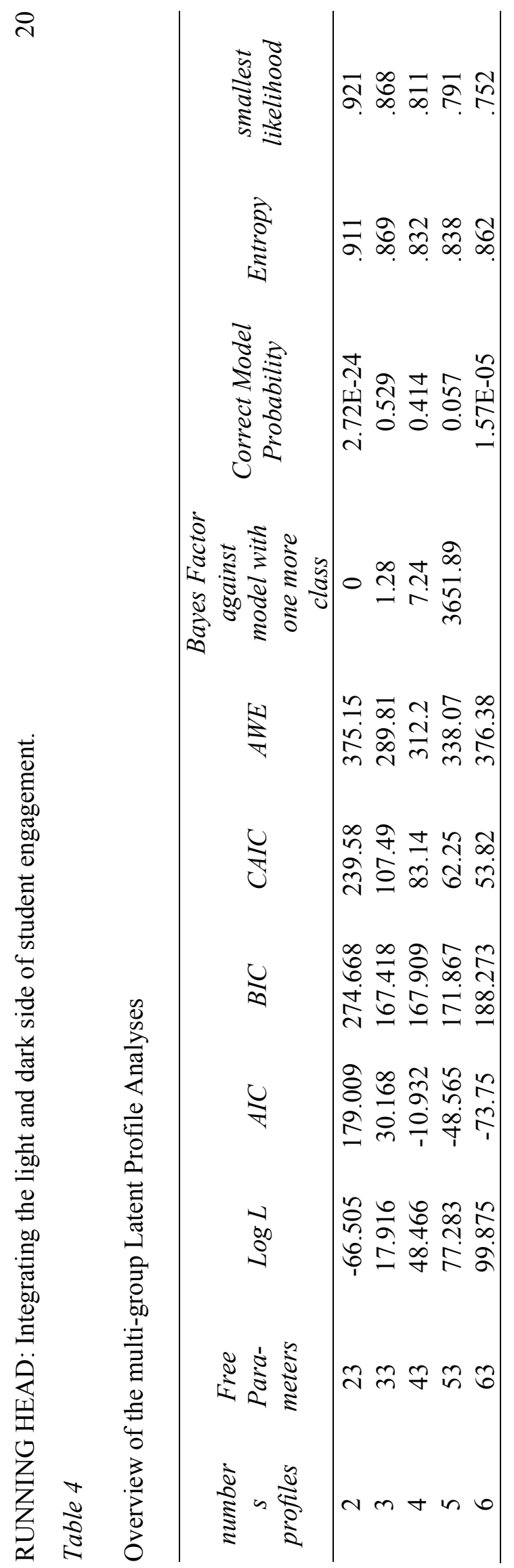


The model with four profiles (Table 5, Figure 1) comprised the two clearly distinct profiles of engaged students (high engagement, low burnout levels) and burned out students (low engagement, high burnout levels). We also found one group of engaged-exhausted students with moderate levels of both engagement and burnout. The fourth profile was characterized by elevated, but still moderate, burnout levels and lower engagement levels, which we interpreted as students at risk for burnout (moderately burned out). The four profile solution shows the importance of combining both light and dark sides of student engagement simultaneously.

\section{Table 5}

Mean scores for engagement and burnout and prevalences of the profiles in the multigroup LPA

\begin{tabular}{|c|c|c|c|c|c|c|c|c|}
\hline & \multicolumn{2}{|c|}{$\begin{array}{c}\text { Engaged } \\
\text { (E) }\end{array}$} & \multicolumn{2}{|c|}{$\begin{array}{c}\text { Engaged- } \\
\text { Exhausted } \\
(E-E)\end{array}$} & \multicolumn{2}{|c|}{$\begin{array}{c}\text { Moderately } \\
\text { Burned out } \\
\text { (M-B) }\end{array}$} & \multicolumn{2}{|c|}{$\begin{array}{c}\text { Burned } \\
\text { Out } \\
\text { (B) }\end{array}$} \\
\hline & $\begin{array}{c}\text { FIN } \\
27.1 \%\end{array}$ & $\begin{array}{c}\text { US } \\
11.5 \%\end{array}$ & $\begin{array}{c}\text { FIN } \\
45.8 \%\end{array}$ & $\begin{array}{c}\text { US } \\
33.3 \%\end{array}$ & $\begin{array}{c}\text { FIN } \\
19.3 \%\end{array}$ & $\begin{array}{c}\text { US } \\
40.7 \%\end{array}$ & $\begin{array}{c}\text { FIN } \\
7.8 \%\end{array}$ & $\begin{array}{c}\text { US } \\
14.4 \\
\%\end{array}$ \\
\hline Engagement (SEI) & .57 & .52 & .51 & .48 & .41 & .36 & .39 & .27 \\
\hline Exhaustion (SBI) & .26 & .15 & .45 & .34 & .59 & .49 & .91 & .84 \\
\hline Cynicism (SBI) & .06 & .08 & .22 & .37 & .52 & .65 & .85 & .86 \\
\hline Inadequacy (SBI) & .13 & .08 & .39 & .32 & .61 & .59 & .92 & .82 \\
\hline
\end{tabular}

Notes. The response scale of SEI and SBI went from 0 to 1 because of the monotonic POMS transformation (Little, 2013).

For profiles identified by LPA, the profiles differed significantly and with strong effect sizes from one another with respect to their indicators (Table 6). The post-hoc t-tests showed that all the profiles differed significantly from each other in burnout indicators. For engagement, in both countries the 'moderately burned out' had lower average engagement than the 'engaged' and the 'engaged-exhausted' and the 'burned-out' group had lower engagement than the 'engaged'. In addition, in the US, the 'engaged-exhausted' had higher average engagement than the 'burned-out'. This implies that the 'engaged-exhausted' did not differ in engagement from the 'engaged' group. 
RUNNING HEAD: Integrating the light and dark side of student engagement.

\section{Table 6}

MANOVA for differences in engagement and burnout between the four profiles in the multi-group LPA

\begin{tabular}{|c|c|c|c|c|c|c|c|c|}
\hline & \multicolumn{2}{|c|}{$F\left(d f_{\text {between }} ; d f_{\text {within }}\right)$} & \multicolumn{2}{|c|}{$p$} & \multicolumn{2}{|c|}{$\eta^{2}$} & \multicolumn{2}{|c|}{$\begin{array}{c}\text { Sign. post-hoc } \\
\text { differences }\end{array}$} \\
\hline & FIN & US & FIN & US & FIN & US & FIN & US \\
\hline Engagement (SEI) & $\begin{array}{c}7.571 \\
(3,212)\end{array}$ & $\begin{array}{c}12.998 \\
(3,247)\end{array}$ & .000 & .000 & .097 & .136 & $\begin{array}{c}\text { E-E\&M-B, } \\
\text { E\&M-B, } \\
\text { E\&B }\end{array}$ & $\begin{array}{c}\text { E-E\&M-B, } \\
\text { E\&M-B, } \\
\text { E\&B, E-E\&B }\end{array}$ \\
\hline Exhaustion (SBI) & $\begin{array}{c}94.400 \\
(3,212)\end{array}$ & $\begin{array}{l}116.474 \\
(3,247)\end{array}$ & .000 & .000 & .572 & .586 & all with all & all with all \\
\hline Cynicism (SBI) & $\begin{array}{l}191.469 \\
(3,212)\end{array}$ & $\begin{array}{l}249.304 \\
(3,247)\end{array}$ & .000 & .000 & .730 & .752 & all with all & all with all \\
\hline Inadequacy (SBI) & $\begin{array}{l}235.662 \\
(3,212)\end{array}$ & $\begin{array}{l}195.671 \\
(3,247)\end{array}$ & .000 & .000 & .769 & .704 & all with all & all with all \\
\hline
\end{tabular}

Notes. The significance level was Bonferroni-corrected.

While the profiles of the four-profile model showed very similar forms in the US and Finland, their prevalences differed. In Finland, 'engaged-exhausted' students formed the biggest group (45.8\%), followed by 'engaged' students (27.1\%). In contrast, students with the 'engaged' profile were rare $(11.5 \%)$ in the US. The 'moderately burned out' group was much more prevalent in the US (40.7\%) than in Finland (19.3\%). 'Burned out' students were rather rare in both countries (7.8\% in Finland, $14.4 \%$ in the U.S.). 


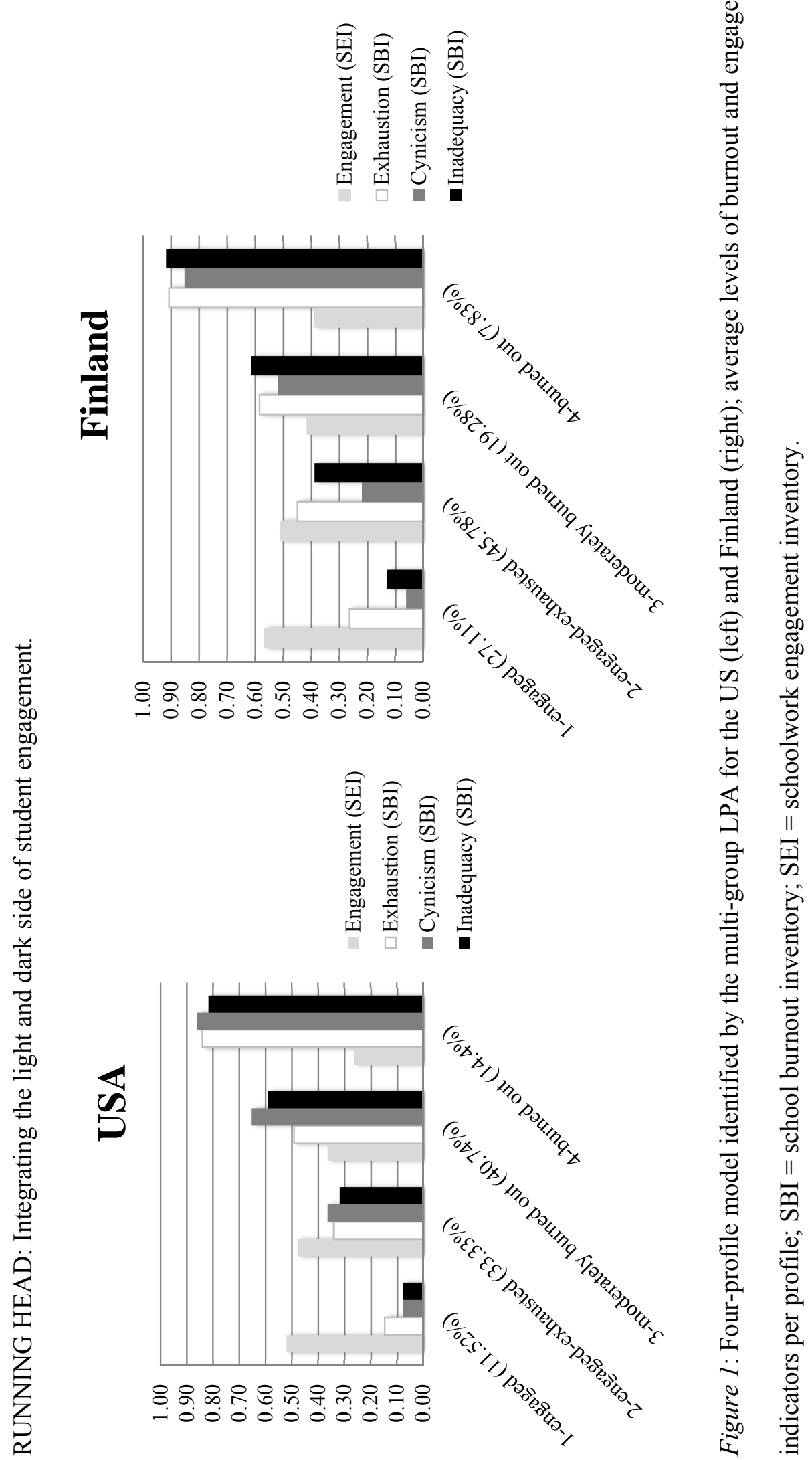


Differences between engagement profiles in average in-school experiences of situational resources, demands, and emotional engagement

Next, we investigated whether the profiles differed with regard to the students' typical experiences of situational demands, resources, and engagement in school. First, for each person, we computed their person-level average (= intra-individual mean) of situational demands, resources and situational engagement across all beeps in school. Then, for each profile, we computed the profile average (= inter-individual mean) of these person-level (intra-individual) means of situational in-school experiences of resources, demands, and engagement. The profile averages were then compared with MANOVAS (Tables 7-8).

In Finland, the profiles differed significantly in their students' average levels of situational demands. Finnish students from the 'burned-out' group experienced higher average levels of situational demands at school than students from the 'engaged' and the 'engaged-exhausted' group. In the US there were no overall profile differences in situational demands. However, there were significant differences between the profiles in the US in situational resources and engagement. The 'engaged' US students experienced higher average levels of situational resources and situational engagement at school than the students in the 'burned out' and the 'moderately burned out' group. In addition, US students in the 'engaged' group reported higher average level of situational engagement at school than those in the 'engaged-exhausted' profile.

\section{Table 7}

Person-level mean scores in ESM in-school experiences of situational demands, resources, and engagement per LPA profile

\begin{tabular}{lcccccccc}
\hline & \multicolumn{2}{c}{$\begin{array}{c}\text { Engaged } \\
\text { (E) }\end{array}$} & \multicolumn{2}{c}{$\begin{array}{c}\text { Engaged-Exhausted } \\
\text { (E-E) }\end{array}$} & $\begin{array}{c}\text { Moderately Burned out } \\
(\mathrm{M}-\mathrm{B})\end{array}$ & \multicolumn{2}{c}{$\begin{array}{c}\text { Burned out } \\
\text { (B) }\end{array}$} \\
& FIN & US & FIN & US & FIN & US & FIN & US \\
\hline Demands & 1.68 & 1.74 & 1.77 & 1.89 & 1.88 & 1.95 & 2.17 & 1.98 \\
Resources & 2.98 & 3.20 & 2.90 & 2.94 & 2.73 & 2.83 & 2.99 & 2.69 \\
Engagement & & & & & & & & \\
& 2.60 & 2.77 & 2.65 & 2.44 & 2.50 & 2.45 & 2.77 & 2.37 \\
\hline
\end{tabular}




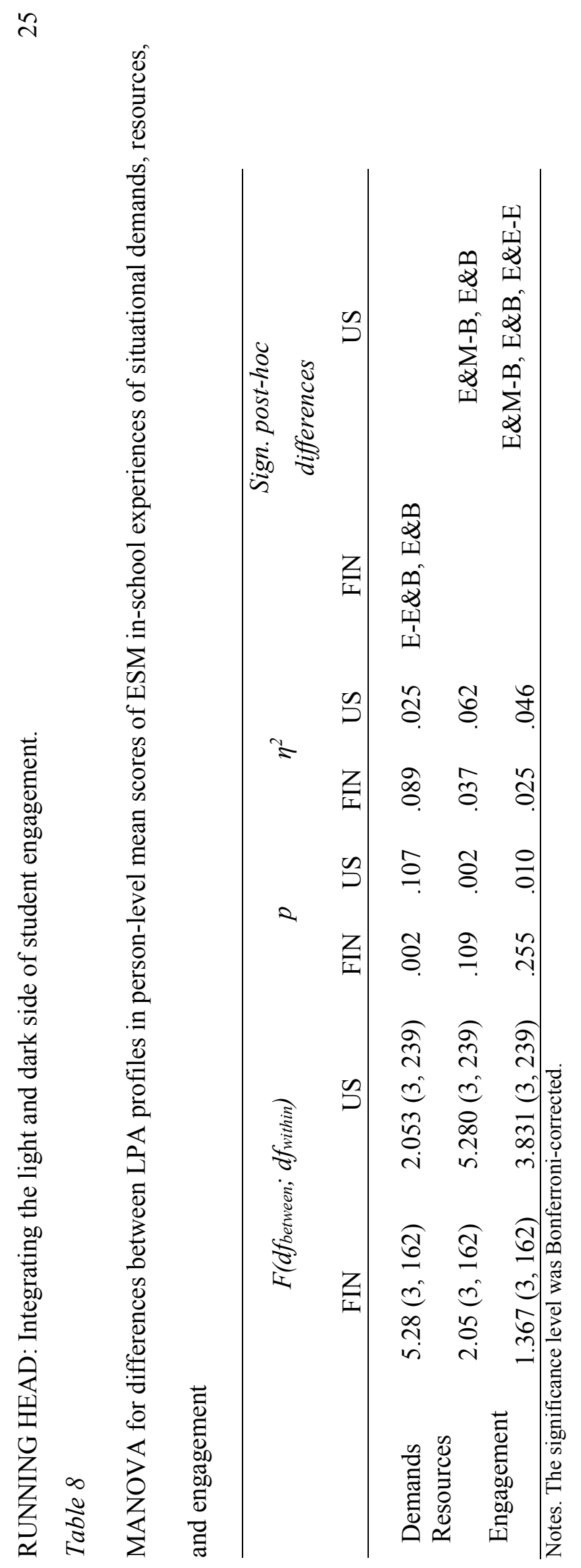




\section{DISCUSSION}

This paper investigated engagement and burnout among Finnish and US high school students by applying three novel approaches to student engagement: First, we combined overall schoolwork engagement and burnout with situational in-school experiences of engagement, demands and resources experienced at school, measured with ESM. Second, we applied a demandsresources model to the school context to describe positive and negative aspects of emotional engagement. Third, we conducted person-oriented analyses to describe subgroups of individuals with different profiles of engagement and burnout and their situational experiences in school. This study aimed at replicating previous findings on the relation between engagement and burnout and their distinct correlations with study demands and study resources (Salmela-Aro \& Upadyaya, 2014), and with previous findings on the co-occurrence of engagement and burnout in the profile of ‘engaged-exhausted students' (Tuominen-Soini \& Salmela-Aro, 2014) within a joint framework.

\subsection{Situational measures of demands, resources, and emotional engagement in school}

Overall levels of engagement and burnout were negatively correlated, which replicated previous findings (e.g. Salmela-Aro \& Upadyaya, 2014). The situational measures of demands, resources, and emotional engagement were reliable and resources experienced at school were related to situational and stable measures of engagement, while demands were related to burnout. This is important and novel finding, as to our knowledge this is the first study to apply a demandsresources perspective to the level of situational school experiences.

The results of the latent profile analyses replicated earlier described profiles of mainly 'engaged', mainly 'burned out' and 'engaged-exhausted' students. The fourth profile of 'moderately burned-out' students resembled the profile labeled 'cynical' (moderate burnout) in Tuominen-Soini \& Salmela-Aro (2014), particularly among the US participants; in the present study, the 'cynicism' level was less pronounced than it was in the earlier study, hence the different label. 
One central finding of this study is that some students experienced elevated engagement and exhaustion simultaneously. These findings from Finland and the US replicate earlier results reported by Tuominen-Soini \& Salmela-Aro (2014). Experiencing this combination of moderate levels of engagement and burnout at the same time can be seen as a risk, in light of the finding by Tuominen-Soini and Salmela-Aro (2014) that 'engaged-exhausted' students were likely to have increased probabilities of developing symptoms of burnout and depression in the long run. This suggests that if a student reports a high or moderate level of engagement, this is not necessarily a positive sign, as it does not necessarily indicate that this student has either had an optimal learning motivation or is not in need of motivational support. Instead, engagement can be exhausting when high effort is maintained over a long period. We believe that students need to learn to maintain and recuperate their resources in order to maintain high levels of engagement over longer time periods without suffering symptoms of exhaustion, burnout and depression.

In view of the predominant emphasis on the positive characteristics of student engagement (Reschly et al., 2008), it would seem important to investigate this 'dark side of student engagement' in more detail in future studies. The identification of a group of engaged-exhausted students is noteworthy in that it points to a very different interpretation from that stemming from the variableoriented analysis of the correlations between school engagement and burnout. The results of the variable-oriented approach suggested that schoolwork engagement was on the whole a positive experience and school burnout a rather negative experience, as indicated here by the negative correlation between the schoolwork engagement (Salmela-Aro \& Upadyaya, 2014) and school burnout (Salmela-Aro et al., 2009), the positive relations between the SEI and situational resources, and the positive relations between the SBI and situational demands. This interpretation of engagement as a generally desirable concept was challenged by the finding of a sizeable group of engaged-exhausted students.

\subsection{Patterns of Engagement Across Countries}


Although the latent profile analyses were conducted separately for the US and Finnish samples, the resulting profiles were very similar. While the overall mean scores suggested that the Finnish students were generally more engaged and less burned out than the US students, the profiles indicated that both samples contained similar groups but with different prevalences. 'Engaged' and 'engaged-exhausted' students were more frequent in the Finnish sample than the US sample, while in the US more students were in the 'burned out' and 'moderately burned out' profiles. This is in line with previous international comparisons in which Finnish students reported relatively high levels of engagement and low levels of burnout compared to students in Belgium, Italy, Germany, the Netherlands and Portugal (Kinnunen et al., in prep.). Interestingly burnout students in Finland are still engaging, they just view these tasks as being more demanding due to the burnout. However, US students who experience burnout see the demand being higher and it is also reflected in their engagement and resources.

The person-oriented profile analyses changed the interpretation from 'Finnish students tend to be more engaged and less burned out than their counterparts in the US' to 'Finland and the US both have quite similar profiles of mainly engaged and mainly burned out students, however the former are more frequent in Finland, while the latter are more frequent in the US'. This is a salutary reminder of the fact that mean scores often do not accurately describe the experience of individuals. This finding can help in identifying students' individual needs for support. Rather than trying to raise the mean score of engagement (or to lower the mean score of burnout) across all students, teachers can and should identify the individual need profiles of their students. Interesting questions for future studies are: Which contextual determinants influence whether students experience mainly engagement, or exhaustive engagement? How can students be challenged and motivated to become more engaged, while also being protected against exhaustion?

\subsection{Practical implications and Limitation}


Teachers need to know what student needs in order to be optimally motivated while being protected against stress and burnout. This study emphasizes how important it is to consider individual students' engagement and burnout levels together to be able to assess students need for support. If a formerly highly motivated student starts to show rather moderate levels of engagement and burnout, it might mean that this student is on the brink of developing a more exhausted, depressed profile, with detrimental effects on her/his subsequent well-being (Tuominen-Soini \& Salmela-Aro, 2014). Describing different groups of students with distinct profiles of engagement and burnout helps identify individual needs for support. In particular students with elevated burnout levels need support to replenish and maintain their resources. Not all disengaged students need higher challenges to enhance their engagement: some are exhausted and need protection against overly challenging demands. An important practical implication is the message that achieving and maintaining high student engagement requires not only interesting and challenging guidance, but also protection against the consequences of over-engagement.

We mostly measured emotional engagement, leaving it to future studies to determine whether similar results follow from more behavioral and cognitive measures of engagement. We used a bi-national sample to test the replicability of our findings, but larger and more representative samples from a larger number of countries would be desirable to examine measurement- and model invariance across countries for both the questionnaire and situational measures, and to check for eventual country-specific influences on correlations, profile forms, and prevalences. Our samples came from just a few schools in specific regions of the US and Finland, and therefore more representative samples are needed before conclusions about the prevalence of particular profiles, or eventual country differences, can be drawn and generalized. While we found four groups resembling those described by Tuominen-Soini \& Salmela-Aro (2014), some of these subgroups were very small. Replications with larger sample sizes are therefore needed. 
The Finnish sample was very homogenous in terms of socioeconomic status and race/ethnic background, but with larger and more diverse samples, the influence of these demographic variables on mean levels, correlations among, and profiles of engagement and burnout could be studied more in detail. Since engagement has many facets (Fredricks et al., 2004) the short situational measures used in this study did not capture engagement as a whole but rather its emotional aspects.

Future studies could employ multilevel modeling with situations nested in individuals to break down the situation-specific and person-specific predictors of engagement and exhaustion. With larger samples multilevel approaches can be combined with latent profile analyses. It should also be noted that in latent profile analysis groups are not observed; instead individuals only resemble the profiles identified by the analysis with a certain probability, and consequently, with a certain measurement error. Future studies could examine group differences in distal outcomes with procedures that take this error in person-profile assignment into account, such as the $\mathrm{BCH}$ method (Asparouhov \& Muthén, 2014).

ESM requires short measures and therefore applies single items as indicators for multifaceted constructs. It is difficult to establish parallel measurement instruments for two countries with single items of this kind, and we cannot rule out the possibility that some of the ESM items assess different experiences. Likewise, the factor structure of the SEI and SBI did not seem equivalent. While we decided to use the same subscales as previous studies, because of our aim to check whether earlier findings would be replicated, future studies should use measures with stronger invariance across the contexts in which they are applied. The fact that the correlations between the situational measures of demands, resources and engagement with the questionnaire measures of school engagement and burnout were relatively similar in both countries might be an indicator of invariance regarding the meaning of the measures across the countries, but this should be tested for both situational and questionnaire measures in future studies. 
The paper has several strengths applying novel person-oriented approach to student engagement by combining overall and situational in-school experiences of engagement, demands and resources and revealed both the light and dark sides of student engagement. 


\section{References}

Asparouhov, T. \& Muthén, B. (2014). Auxiliary variables in mixture modeling: Using the BCH method in Mplus to estimate a distal outcome model and an arbitrary second model.

Bakker, A. B. \& Demerouti, E. (2007). The Job Demands-Resources model: state of the art. Journal of Managerial Psychology, 22(3), 309 - 328.

Bergman, L. R., \& Magnusson, D. (1997). A person-oriented approach in research on developmental psychopathology. Development and Psychopathology, 9, 291-319.

Csikszentmihalyi, M., \& Csikszentmihalyi, I. (1988). Optimal experience: Psychological studies of Flow in consciousness. Cambridge: University Press.

Engeser, S. \& Rheinberg, F. (2008). Flow, moderators of challenge-skill-balance and performance. Motivation and Emotion, 32, 158-172.

Finn, J. D. (1989). Withdrawing from school. Review of Educational Research, 59, 117-142.

Fredricks \& McColskey (2012). The Measurement of Student Engagement: A Comparative Analysis of Various Methods and Student Self-report Instruments. In: S. L. Christenson, A. L. Reschly, \& C. Wylie (Eds.). Handbook of Research on Student Engagement (pp.763-782), Springer, New York,

Fredricks, J. A., Blumenfeld, P. C. \& Paris, A. H. (2004). School Engagement: Potential of the Concept, State of the Evidence. Review of Educational Research, 74(1), 59-109.

Goetz, T., Bieg, M., Lüdtke, O., Pekrun, R., \& Hall, N. C., (2013). Do girls really experience more anxiety in mathematics? Psychological Science, 24, 2079-2087.

Jimerson, S. R., Campos, E. \& Greif, J. L. (2003). Toward an Understanding of Definitions and Measures of School Engagement and Related Terms. The California School Psychologist, 8, $7-28$.

Linnakylä, P. \& Malin, A. 2008. Finnish Students' School Engagement Profiles in the Light of PISA 2003. Scandinavian Journal of Educational Research, 52 (6), 583-602. 
RUNNING HEAD: Integrating the light and dark side of student engagement.

Little, T. D. (2013). Longitudinal Structural Equation Modeling (Methodology in the Social Sciences). The Guilford Press.

Masyn, K. (2013). Latent class analysis and finite mixture modeling. In T. D. Little (Ed.) The Oxford handbook of quantitative methods in psychology (Vol. 2, pp. 551-611). New York, NY: Oxford University Press.

Muthén, L.K. and Muthén, B.O. (1998-2012). Mplus User's Guide. Seventh Edition. Los Angeles, CA: Muthén \& Muthén..

OECD. (2013). PISA 2012 results: Ready to learn: Students' engagement, drive, and self-beliefs (Volume III). Paris, France: OECD Publishing.

Pekrun, R., Elliot, A. J., \& Maier, M. A. (2009). Achievement Goals and Achievement Emotions: Testing a Model of Their Joint Relations With Academic Performance. Journal of Educational Psychology, 101(1), 115-135.

Petrou, P., Demerouti, E., Peeters, M.C.W., Schaufeli, W.B. \& Hetland, J. (2012). Crafting a job on a daily basis: contextual correlates and the link to work engagement. Journal of Organizational Behavior, 33(8), 1120-1141.

Ram, N. \& Grimm, K. (2009). Growth Mixture Modeling: A method for identifying differences in longitudinal change among unobserved groups. International Journal of Behavioral Development, 33, 565-576.

Reizle, M. (2013). Introduction: Doubts and insights concerning variable- and person-oriented approaches to human development. European Journal of Developmental Psychology, 10(1), $1-8$.

Reschly, A. L., Huebner, E. S., Appleton, J. J., Antaramian, S. (2008). Engagement as flourishing: The contribution of positive emotions and coping to adolescents' engagement at school and with learning. Psychology in the Schools, 45(5), 419-431. 
Roorda, D. L., Koomen, H. M. Y. Spilt, J. L., Oort, F. J. (2011). The Influence of Affective Teacher-Student Relationships on Students' School Engagement and Achievement: A Meta-Analytic Approach. Review of Educational Research, 81(4), 493-529.

Rost, J. (2006). Latent-Class Analyse. In: F. Petermann \& M. Eid (Eds). Handbuch der Psychologischen Diagnostik (pp. 275-287). Göttingen: Hogrefe.

Salmela-Aro, Kiuru, N., Leskinen, E., \& Nurmi, J. E. (2009a). School-Burnout inventory (SBI). Reliability and validity. European Journal of Psychological Assessment, 25(1), 48-57.

Salmela-Aro, K., Savolainen, H., Holopainen, L. (2009b). Depressive Symptoms and School Burnout During Adolescence: Evidence from Two Cross-lagged Longitudinal Studies. Journal of Youth and Adolescence, 38 (10), 1316-1327.

Salmela-Aro, K., \& Upadaya, K. (2012). The Schoolwork Engagement Inventory. European Journal of Psychological Assessment, 28(1), 60-67.

Salmela-Aro, K., \& Upadyaya, K. (2014). School burnout and engagement in the context of demands-resources model. British Journal of Educational Psychology, 84(1), 137-151.

Schneider, B., Krajic, J., Lavonen, J., Salmela-Aro, K. et al (in press). Investigating optimal learning moments in U.S. and Finnish science classes. Journal of Research in ScienceTeaching.

Shernoff, D. J. \& Vandell, D. B. (2007). Engagement in after-school program activities: quality of experience from the perspective of participants. Journal of Youth and Adolescence, 36, 891903.

Simbula, S. (2010). Daily fluctuations in teachers' well-being: A diary study using the Job Demands-Resources model. Anxiety, Stress \& Coping: An International Journal, 23, $563-$ 584. 
RUNNING HEAD: Integrating the light and dark side of student engagement.

Skinner, E.,Furrer, C., Marchand, G., \& Kinderman, T. (2008). Engagement and Disaffection in the classroom: Part of a larger Motivational Dynamic? Journal of educational Psychology, 100(4), 765-781.

Tuominen-Soini, H., \& Salmela-Aro, K. (2014). Schoolwork engagement and burnout among Finnish high school students and young adults: Profiles, progressions and educational outcomes. Developmental Psychology, 50(3), 649-662.

Tynkkynen, L., \& Salmela-Aro, K. (2012). Gendered pathways in school burnout among adolescents. Journal of Adolescence, 35 (4), 929-939.

Voelkl, K. E. (1997). Identification with school. American Journal of Education, 105, 204-319.

Wang, M.-T. \& Degol, J. (2013). Motivational pathways to STEM career choices: Using expectancy-value perspective to understand individual and gender differences in STEM fields. Developmental Review 33, 304-340.

Wang, M.-T., \& Peck, S. (2013). Adolescent educational success and mental health vary across school engagement profiles. Developmental Psychology, 49, 1266-1276. 


\section{APPENDIX}

\section{Table A-1}

Inter-correlations of situational experiences of resources, demands, and engagement in school (within-level = above the diagonal; between-level = below the diagonal)

\begin{tabular}{|c|c|c|c|c|c|c|}
\hline & \multicolumn{2}{|c|}{ Resources } & \multicolumn{2}{|c|}{ Demands } & \multicolumn{2}{|c|}{ Engagement } \\
\hline & US & FIN & US & FIN & US & FIN \\
\hline Resources & - & - & $-.398 *$ & $-.433 *$ & $.607 *$ & $.689 *$ \\
\hline Demands & -.352 & $-.214^{*}$ & - & - & $-.144 *$ & $-.295 *$ \\
\hline Engagement & $.633^{*}$ & $.727 *$ & .031 & .028 & - & - \\
\hline
\end{tabular}


RUNNING HEAD: Integrating the light and dark side of student engagement.

Table A-2

Descriptives for ESM experiences in school

\begin{tabular}{|c|c|c|c|c|c|c|c|c|}
\hline & \multicolumn{2}{|c|}{ Mbetween } & \multicolumn{2}{|c|}{ Variance within } & \multicolumn{2}{|c|}{ Variancebetween } & \multicolumn{2}{|c|}{$I C C$} \\
\hline & US & Fin & US & Fin & US & Fin & US & Fin \\
\hline Resources & 2.889 & 2.910 & .306 & .139 & .255 & .178 & .443 & .375 \\
\hline Demands & 1.916 & 1.801 & .213 & .168 & .093 & .041 & .387 & .334 \\
\hline Engagement & 2.462 & 2.628 & .080 & .126 & .159 & .126 & .351 & .254 \\
\hline
\end{tabular}

